\title{
Tangible and Intangible Attributes for the Development of Convention Tourism - An Economic Proposition
}

\author{
A. Lajwanti Naidu, Manohar Sajnani, Zeenat Zaidi
}

\begin{abstract}
The present working paper documents the results of a pilot study on the Economic Impacts of Development of Convention Tourism in Hyderabad. Gaining impetus from various stakeholders of the tourism industry, the research employs the importance - performance model. The assessment is done under two heads: tangibles and intangibles, to examine whether the city of Hyderabad does live up to its reputation of being the "Convention Capital of India" attracting, in particular, leading industry giants to participate in congresses and conventions. The main study would endeavor to provide a sustainable business model to supplement the efforts of Government of Andhra Pradesh.
\end{abstract}

Keywords-Convention Tourism, Economic Proposition, Importance Performance Model.

\section{INTRODUCTION}

It has been well recognized that key to economic development is the growth of the corporate sector. Without entering into questions of value judgments, which is best left to the Legislature concerned, the effort is to create conducive atmosphere for greatest value addition. The means by which the corporate devise new products and services, or to improve their quality depends on their systems to gather data and analyze them to arrive at definite conclusions. These are then to be replicated in several markets. Typically, expansion plans require outreach to those who can be impressed with business ideas. Conferences, conventions and meetings play a very important role in attracting investments, in new areas, fields and markets.

High-end and niche products and services are known to account for over $80 \%$ of the growth in the Gross Value Addition in developed economies. New products and models are unveiled and launched in larger convention spaces. Business Tourism includes facilitating organization of such conferences, conventions and meetings, blending them with the accumulated knowledge and experience of various cultures and geographies, making it a pleasant and productive experience, besides the purely business motive.

Revised Manuscript Received on September 22, 2019.

A.Lajwanti Naidu, Amity University,Noida ,Uttar Pradesh .Email: lajwantinaidu201@gmail.com

Prof Dr ManoharSajnani. (Dean), Hospitality and Tourism, Amity University ,Noida ,UttarPradesh,India,

Dr. ZeenatZaidi (Principal), Govt Degree College, B.B. Nagar, Uttar Pradesh.
Mumbai is considered to be the traditional economic capital of India. The development of Mumbai was primarily owing to its proximity to the huge natural port, which facilitated merchandise trade. However, the times have now changed, with business revolving more on computer science, esp software/ applications ("App" for short) and e-commerce, for which the hotspots are Hyderabad and Bangalore, which now contest with Mumbai and other cities for their economic space. The Conventions are not confined to the software/ computer-based products; rather encompass even furniture (timber), and food processing, the meeting point of the primary and secondary sectors of the economy, and cars (automobiles), glass (construction sector), energy (conventional and non-conventional sources). Even marriages and spiritual meetings at convention centers are not unknown.

The state-of-the-art Hyderabad International Convention Center (HICC) is the largest in India, hence there is a need to evaluate its economic potential and impact today.

\section{LITERATURE REVIEW}

Baun, B. M., \& Rungeling, B.in (1992) justifies that the regional economy mainly depends on the existence of the Convention Bureaus. The Study shows that Convention and Meeting Industry is highly beneficial which can boost the seasonal fluctuations and also enhance the source of economy in tourism activity. The case of Orlando Florida indicatedand proved that the Conventions have facilitated the economical growth of the country.Braun, B. M.in(1992)examined the economic impact of various conventions on cities. The study used various local convention characteristics to adjust national and International data. It also probed into two types of spending that is attending and holding a Convention done by the delegate before and after the Convention. The study was then analyzed with the help of Input output model which had direct Effect and Multipliers measured in terms of wages and employment.Kim, S. S., Chon, K., \& Chung, K. Y.in (2003) observes in their study that Convention Tourism had an economic impact. The analysis was done by the input outputmodel and the subsequent multiplier effect.

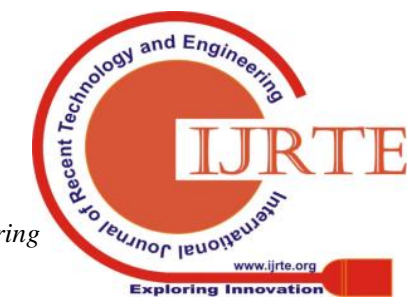


This model was used to investigate the Economic Impact with various variables as Employment ,income obtained at the convention center, rate of Exchange earnings value added imposer and other substitution Effects, According to the final results of the Study it is estimated that economic Impact is very significant to the Korean Economy. Yoo, J. J. E., \& Weber, K.in (2005) suggested future directions of research with the aim of identifying focus areas. The main purpose of the study was to determine the progress in the Convention Tourism by utilizing the content published in 14 academic journals. The study proved that despite its economic significance and rapid growth rate very little research is done in the specified area.Dwyer, L., Mellor, R., Mistilis, N., \& Mules, T. in (2000)focused in their article on the tangible and intangible impacts of the Conventions and events. Most of the time Governments and Public offices are asked to provide financial assistance for any special events. But the major concern is what kind of assistance is required. The study Cleary indicated that the perceived frame work ofanalysis can be used to determine which event should support funds. In the year 1998 Tourism New south wales has commissioned the development of supportive frame work for assessing the economic impact of events and convention industry. This given framework was expected to serve as a device for discerning global trends in economic impacts of events and conventions, Apart from it also serve as a guide to projecting the likely economic impacts of future events and conventions by type and by location. Dwyer, Lin (2002)highlightsissues which are relevant in estimating the economic contribution of the convention industry. There are various steps involved in economic impact assessment of the convention sector from the perspective of the host destination, namely: (1) estimation of the in-scope expenditure of convention participants and accompanying persons; (2) estimation of the in-scope expenditure of convention organizers, associations, and sponsors; (3) allocation of total in-scope expenditure to particular industries; and (4) application of multipliers to total in-scope expenditure to estimate the contribution to regional income and gross regional product. Conceptual and empirical issues pertinent to each step are discussed, and gaps in the research literature are identified.

\section{OBJECTIVE OF THE STUDY}

The Objective of the study is to assess the economic impact of Convention Tourism as a Developmental Facility. This will be achieved in two steps, by examining the present status of convention tourism in Hyderabad, and by exploring the strengths and unique features of local culture that might suit the development of responsible business tourism.

\section{SCOPE OF THE STUDY}

Convention Tourism is worldwide have been succeeded because of a deliberate effort to promote destinations. It has been observed that the most of the European and North American convention centers have been enormously funded by the Governmental bodies. Apart from this the past experiences prove that Countries like Singapore and Thailand indicate that public private partnership works very well. The present study is conducted to understand the functioning of the Hyderabad International Convention Center Position and Contribution to the states exchequer.

\section{RESEARCH METHODOLOGY}

The present study is contemporary in nature and is also working paper for the doctoral work. Extensive Secondary data was consulted for the study based on the large number of Sources including a mix of Primary and Secondary sources. An Instrument which was based on Importance Performance Model was administered at leading Travel show at Hyderabad. Primary sources will comprise of Consumer Survey, Conference Visitors, Conference Organizers, and Personal Interview with the Stake Holders in the Industry. Secondary Sources include the Industry Report from International Association for Convention VisitorBureau, Journal Articles, Professional Journal and others.

The key observations may be taken from the Ministry of Tourism International Passenger Survey IPA and Tourism Satellite Account. TSA, 2016 Global meetings and Events forecast.These studies will give an insight into the latest trends of Business Traveler and its direct and indirect Impact on Convention Tourism.

Sample and Sample Techniques.A sample of 48 questionnaires were taken from the demographic region of India from major conventions centers in Kolkata /Mumbai, Delhi and Hyderabad. The sampling technique was simple random sampling and the data analysis was done in $\mathrm{R}$ software. Primary data was collected with a questionnaire with 34 attributes.

\section{Analysis and Interpretations:}

The data for pre-testing was collected across Various Convention Centers at Hyderabad. A total of forty eight respondents, belonging to India and ten other countries answered the questions. The Age-wise and gender-wise distribution is as follows: 
Table 1.Age wise and gender-wise distribution

\begin{tabular}{|c|c|c|c|c|c|c|c|c|c|}
\hline & \multirow{2}{*}{$\begin{array}{l}y \\
\frac{y}{\varepsilon} \\
0 \\
0 \\
0 \\
\alpha\end{array}$} & \multirow{2}{*}{ 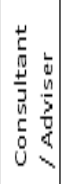 } & \multirow{2}{*}{$\begin{array}{ll}0 & \\
\varepsilon & \\
E & \overline{5} \\
0 & 0 \\
0 & \vdots \\
0 & \bar{c}\end{array}$} & \multirow{2}{*}{ 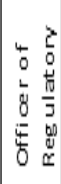 } & \multicolumn{4}{|c|}{ Private commercial enterprise } & \multirow{2}{*}{ Total } \\
\hline & & & & & $\begin{array}{l}\text { High Level } \\
\text { Management }\end{array}$ & \begin{tabular}{|l|} 
Middle Level \\
Management
\end{tabular} & $\begin{array}{l}\text { Operational } \\
\text { Staff }\end{array}$ & Others & \\
\hline American & 0 & 0 & 1 & 0 & 0 & 0 & 0 & 0 & 1 \\
\hline Bangladeshi & 0 & 0 & 0 & 4 & 0 & 0 & 0 & 0 & 4 \\
\hline Bhutanese & 0 & 0 & 0 & 0 & 0 & 0 & 1 & 0 & 1 \\
\hline Chinese & 0 & 0 & 0 & 0 & 0 & 2 & 0 & 0 & 2 \\
\hline Emarati & 0 & 0 & 0 & 0 & 1 & 0 & 0 & 0 & 1 \\
\hline Indian & 4 & 2 & 3 & 5 & 1 & 5 & 7 & 0 & 27 \\
\hline KOREAN & 0 & 0 & 0 & 1 & 0 & 0 & 0 & 0 & 1 \\
\hline Kenyan & 0 & 0 & 0 & 2 & 5 & 0 & 0 & 0 & 7 \\
\hline NEPALI & 0 & 0 & 0 & 1 & 0 & 0 & 0 & 0 & 1 \\
\hline Russian & 0 & 0 & 0 & 1 & 0 & 0 & 1 & 0 & 2 \\
\hline $\begin{array}{l}\text { South } \\
\text { African }\end{array}$ & 0 & 0 & 0 & 1 & 0 & 0 & 0 & 0 & 1 \\
\hline Total & 0 & 1 & 2 & 12 & 1 & 7 & 9 & 2 & 48 \\
\hline
\end{tabular}

Table 2. Nationality-wise gender-wise distribution

\begin{tabular}{|l|r|r|r|}
\hline Nationality & \multicolumn{1}{|l|}{ Female } & \multicolumn{1}{|l|}{ Male } & \multicolumn{1}{l|}{ Total } \\
\hline American & 0 & 1 & $\mathbf{1}$ \\
\hline Bangladeshi & 1 & 3 & $\mathbf{4}$ \\
\hline Bhutanese & 0 & 1 & $\mathbf{1}$ \\
\hline Chinese & 0 & 2 & $\mathbf{2}$ \\
\hline Emarati & 0 & 1 & $\mathbf{1}$ \\
\hline Indian & 6 & 21 & $\mathbf{2 7}$ \\
\hline Korean & 0 & 1 & $\mathbf{1}$ \\
\hline Kenyan & 2 & 5 & $\mathbf{7}$ \\
\hline Nepali & 1 & 0 & $\mathbf{1}$ \\
\hline Russian & 0 & 2 & $\mathbf{2}$ \\
\hline South African & 1 & 0 & $\mathbf{1}$ \\
\hline & & & \\
\hline Total & $\mathbf{1 1}$ & $\mathbf{3 7}$ & $\mathbf{4 8}$ \\
\hline
\end{tabular}

The Respondents were from varied backgrounds, as may be seen from the different kinds of organizations to which they belonged:

Table 3. Different kinds of organizations

Table4. The Economic Sector-wise distribution of the respondents is as follows:

\begin{tabular}{|l|r|r|r|}
\hline Age-group & \multicolumn{1}{|l|}{ Female } & \multicolumn{1}{|l|}{ Male } & \multicolumn{1}{|l|}{ Total } \\
\hline $21-30$ & 1 & 8 & 9 \\
\hline $31-40$ & 6 & 13 & 19 \\
\hline $41-60$ & 4 & 16 & 20 \\
\hline Total & 11 & 28 & 48 \\
\hline
\end{tabular}

Table 5.The capacity in which the various respondents had come to the event were:

\begin{tabular}{|l|r|r|r|r|r|r|r|}
\hline & JOURNALIST & Mentor & Organizer & Participant & Speaker & Sponsor & \multicolumn{1}{c|}{ Total } \\
\hline American & 0 & 0 & 0 & 0 & 0 & 1 & 1 \\
\hline $\begin{array}{l}\text { Bangladesh } \\
\text { i }\end{array}$ & 2 & 1 & 0 & 0 & 0 & 1 & 4 \\
\hline Bhutanese & 0 & 0 & 0 & 0 & 0 & 1 & 1 \\
\hline Chinese & 1 & 0 & 0 & 0 & 0 & 1 & 2 \\
\hline Emarati & 0 & 0 & 0 & 1 & 0 & 0 & 1 \\
\hline Indian & 4 & 5 & 1 & 5 & 1 & 11 & 27 \\
\hline KOREAN & 0 & 0 & 0 & 0 & 0 & 1 & 1 \\
\hline Kenyan & 0 & 0 & 1 & 0 & 0 & 0 & 1 \\
\hline NEPALI & 0 & 0 & 0 & 0 & 0 & 1 & 1 \\
\hline Russian & 2 & 0 & 0 & 0 & 0 & 0 & 2 \\
\hline Total & 9 & 1 & 1 & 5 & 1 & 17 & 48 \\
\hline
\end{tabular}

Table 6.The Response Matrix for each question is as follows:

\begin{tabular}{|c|c|c|c|c|c|c|}
\hline \multirow{2}{*}{$\begin{array}{l}\text { Questio } \\
\mathrm{n}\end{array}$} & \multirow{2}{*}{ Importance } & \multicolumn{4}{|c|}{ Performance } & \multirow{2}{*}{ Total } \\
\hline & & High & Low & Medium & NA & \\
\hline (1) & (2) & (3) & (4) & (5) & (6) & (7) \\
\hline \multirow[t]{5}{*}{1} & High & 10 & 0 & 2 & 0 & 12 \\
\hline & Low & 0 & 0 & 0 & 0 & 0 \\
\hline & Medium & 0 & 0 & 0 & 0 & 0 \\
\hline & NA & 0 & 0 & 0 & 22 & 22 \\
\hline & Total & 10 & 0 & 2 & 22 & 34 \\
\hline \multirow[t]{5}{*}{2} & High & 10 & 0 & 2 & 0 & 12 \\
\hline & Low & 0 & 0 & 0 & 0 & 0 \\
\hline & Medium & 0 & 0 & 0 & 0 & 0 \\
\hline & NA & 0 & 0 & 0 & 22 & 22 \\
\hline & Total & 10 & 0 & 2 & 22 & 34 \\
\hline \multirow[t]{5}{*}{3} & High & 9 & 0 & 2 & 0 & 11 \\
\hline & Low & 0 & 0 & 0 & 0 & 0 \\
\hline & Medium & 1 & 0 & 0 & 0 & 1 \\
\hline & NA & 0 & 0 & 0 & 22 & 22 \\
\hline & Total & 10 & 0 & 2 & 22 & 34 \\
\hline \multirow[t]{4}{*}{4} & High & 9 & 0 & 2 & 0 & 11 \\
\hline & Low & 0 & 1 & 0 & 0 & 1 \\
\hline & Medium & 0 & 0 & 0 & 0 & 0 \\
\hline & NA & 0 & 0 & 0 & 22 & 22 \\
\hline
\end{tabular}


Tangible and Intangible Attributes for the Development of Convention Tourism - An Economic Proposition

\begin{tabular}{|c|c|c|c|c|c|c|}
\hline \multirow{2}{*}{$\begin{array}{l}\text { Questio } \\
\mathrm{n}\end{array}$} & \multirow{2}{*}{ Importance } & \multicolumn{4}{|c|}{ Performance } & \multirow{2}{*}{ Total } \\
\hline & & High & Low & Medium & NA & \\
\hline \multirow[t]{2}{*}{ (1) } & (2) & (3) & (4) & (5) & (6) & (7) \\
\hline & Total & 9 & 1 & 2 & 22 & 34 \\
\hline \multirow[t]{5}{*}{5} & High & 19 & 0 & 8 & 0 & 27 \\
\hline & Low & 0 & 5 & 0 & 0 & 5 \\
\hline & Medium & 0 & 0 & 2 & 0 & 2 \\
\hline & NA & 0 & 0 & 0 & 0 & 0 \\
\hline & Total & 19 & 5 & 10 & 0 & 34 \\
\hline \multirow[t]{5}{*}{6} & High & 18 & 0 & 4 & 0 & 22 \\
\hline & Low & 0 & 0 & 0 & 0 & 0 \\
\hline & Medium & 2 & 0 & 10 & 0 & 12 \\
\hline & NA & 0 & 0 & 0 & 0 & 0 \\
\hline & Total & 20 & 0 & 14 & 0 & 34 \\
\hline \multirow[t]{5}{*}{7} & High & 9 & 2 & 7 & 0 & 18 \\
\hline & Low & 0 & 3 & 0 & 0 & 3 \\
\hline & Medium & 1 & 0 & 12 & 0 & 13 \\
\hline & NA & 0 & 0 & 0 & 0 & 0 \\
\hline & Total & 10 & 5 & 19 & 0 & 34 \\
\hline \multirow[t]{5}{*}{8} & High & 7 & 2 & 12 & 0 & 21 \\
\hline & Low & 0 & 1 & 1 & 0 & 2 \\
\hline & Medium & 1 & 0 & 10 & 0 & 11 \\
\hline & NA & 0 & 0 & 0 & 0 & 0 \\
\hline & Total & 8 & 3 & 23 & 0 & 34 \\
\hline \multirow[t]{5}{*}{9} & High & 10 & 2 & 11 & 0 & 23 \\
\hline & Low & 0 & 3 & 0 & 0 & 3 \\
\hline & Medium & 0 & 1 & 7 & 0 & 8 \\
\hline & NA & 0 & 0 & 0 & 0 & 0 \\
\hline & Total & 10 & 6 & 18 & 0 & 34 \\
\hline \multirow[t]{5}{*}{10} & High & 11 & 4 & 11 & 0 & 26 \\
\hline & Low & 0 & 3 & 0 & 0 & 3 \\
\hline & Medium & 1 & 1 & 3 & 0 & 5 \\
\hline & NA & 0 & 0 & 0 & 0 & 0 \\
\hline & Total & 12 & 8 & 14 & 0 & 34 \\
\hline \multirow[t]{5}{*}{11} & High & 12 & 2 & 12 & 0 & 26 \\
\hline & Low & 0 & 4 & 0 & 0 & 4 \\
\hline & Medium & 1 & 0 & 3 & 0 & 4 \\
\hline & NA & 0 & 0 & 0 & 0 & 0 \\
\hline & Total & 13 & 6 & 15 & 0 & 34 \\
\hline \multirow[t]{5}{*}{12} & High & 16 & 0 & 7 & 0 & 23 \\
\hline & Low & 0 & 5 & 0 & 0 & 5 \\
\hline & Medium & 2 & 1 & 3 & 0 & 6 \\
\hline & NA & 0 & 0 & 0 & 0 & 0 \\
\hline & Total & 18 & 6 & 10 & 0 & 34 \\
\hline \multirow[t]{3}{*}{13} & High & 11 & 0 & 9 & 0 & 20 \\
\hline & Low & 0 & 4 & 0 & 0 & 4 \\
\hline & Medium & 1 & 1 & 8 & 0 & 10 \\
\hline
\end{tabular}

\begin{tabular}{|c|c|c|c|c|c|c|}
\hline \multirow{2}{*}{$\begin{array}{l}\text { Questio } \\
\mathrm{n}\end{array}$} & \multirow{2}{*}{ Importance } & \multicolumn{4}{|c|}{ Performance } & \multirow{2}{*}{ Total } \\
\hline & & High & Low & Medium & NA & \\
\hline \multirow[t]{3}{*}{ (1) } & (2) & (3) & (4) & (5) & (6) & (7) \\
\hline & NA & 0 & 0 & 0 & 0 & 0 \\
\hline & Total & 12 & 5 & 17 & 0 & 34 \\
\hline \multirow[t]{5}{*}{14} & High & 7 & 1 & 14 & 0 & 22 \\
\hline & Low & 0 & 2 & 0 & 0 & 2 \\
\hline & Medium & 2 & 0 & 8 & 0 & 10 \\
\hline & NA & 0 & 0 & 0 & 0 & 0 \\
\hline & Total & 9 & 3 & 22 & 0 & 34 \\
\hline \multirow[t]{5}{*}{15} & High & 12 & 1 & 11 & 0 & 24 \\
\hline & Low & 0 & 4 & 0 & 0 & 4 \\
\hline & Medium & 1 & 0 & 5 & 0 & 6 \\
\hline & NA & 0 & 0 & 0 & 0 & 0 \\
\hline & Total & 13 & 5 & 16 & 0 & 34 \\
\hline \multirow[t]{5}{*}{16} & High & 13 & 1 & 10 & 0 & 24 \\
\hline & Low & 0 & 3 & 0 & 0 & 3 \\
\hline & Medium & 1 & 0 & 6 & 0 & 7 \\
\hline & NA & 0 & 0 & 0 & 0 & 0 \\
\hline & Total & 14 & 4 & 16 & 0 & 34 \\
\hline \multirow[t]{5}{*}{17} & High & 14 & 2 & 9 & 0 & 25 \\
\hline & Low & 0 & 2 & 0 & 0 & 2 \\
\hline & Medium & 1 & 1 & 5 & 0 & 7 \\
\hline & NA & 0 & 0 & 0 & 0 & 0 \\
\hline & Total & 15 & 5 & 14 & 0 & 34 \\
\hline \multirow[t]{5}{*}{18} & High & 11 & 2 & 10 & 0 & 23 \\
\hline & Low & 1 & 3 & 0 & 0 & 4 \\
\hline & Medium & 1 & 1 & 5 & 0 & 7 \\
\hline & NA & 0 & 0 & 0 & 0 & 0 \\
\hline & Total & 13 & 6 & 15 & 0 & 34 \\
\hline \multirow[t]{5}{*}{19} & High & 20 & 1 & 8 & 0 & 29 \\
\hline & Low & 0 & 2 & 0 & 0 & 2 \\
\hline & Medium & 0 & 1 & 2 & 0 & 3 \\
\hline & NA & 0 & 0 & 0 & 0 & 0 \\
\hline & Total & 20 & 4 & 10 & 0 & 34 \\
\hline \multirow[t]{5}{*}{20} & High & 18 & 1 & 5 & 0 & 24 \\
\hline & Low & 0 & 2 & 0 & 0 & 2 \\
\hline & Medium & 2 & 0 & 6 & 0 & 8 \\
\hline & NA & 0 & 0 & 0 & 0 & 0 \\
\hline & Total & 20 & 3 & 11 & 0 & 34 \\
\hline \multirow[t]{5}{*}{21} & High & 14 & 1 & 11 & 0 & 26 \\
\hline & Low & 0 & 2 & 0 & 0 & 2 \\
\hline & Medium & 2 & 0 & 4 & 0 & 6 \\
\hline & NA & 0 & 0 & 0 & 0 & 0 \\
\hline & Total & 16 & 3 & 15 & 0 & 34 \\
\hline
\end{tabular}


International Journal of Recent Technology and Engineering (IJRTE) ISSN: 2277-3878, Volume-8 Issue-2S10, September 2019

\begin{tabular}{|c|c|c|c|c|c|c|}
\hline \multirow{2}{*}{$\begin{array}{l}\text { Questio } \\
\mathrm{n}\end{array}$} & \multirow{2}{*}{ Importance } & \multicolumn{4}{|c|}{ Performance } & \multirow{2}{*}{ Total } \\
\hline & & High & Low & Medium & NA & \\
\hline (1) & (2) & (3) & (4) & (5) & (6) & (7) \\
\hline \multirow[t]{5}{*}{22} & High & 18 & 1 & 8 & 0 & 27 \\
\hline & Low & 0 & 1 & 0 & 0 & 1 \\
\hline & Medium & 1 & 0 & 5 & 0 & 6 \\
\hline & NA & 0 & 0 & 0 & 0 & 0 \\
\hline & Total & 19 & 2 & 13 & 0 & 34 \\
\hline \multirow[t]{5}{*}{23} & High & 21 & 2 & 6 & 0 & 29 \\
\hline & Low & 0 & 1 & 0 & 0 & 1 \\
\hline & Medium & 1 & 0 & 3 & 0 & 4 \\
\hline & NA & 0 & 0 & 0 & 0 & 0 \\
\hline & Total & 22 & 3 & 9 & 0 & 34 \\
\hline \multirow[t]{5}{*}{24} & High & 14 & 3 & 8 & 0 & 25 \\
\hline & Low & 0 & 2 & 0 & 0 & 2 \\
\hline & Medium & 0 & 2 & 5 & 0 & 7 \\
\hline & NA & 0 & 0 & 0 & 0 & 0 \\
\hline & Total & 14 & 7 & 13 & 0 & 34 \\
\hline \multirow[t]{5}{*}{25} & High & 14 & 1 & 9 & 0 & 24 \\
\hline & Low & 0 & 1 & 0 & 0 & 1 \\
\hline & Medium & 2 & 1 & 6 & 0 & 9 \\
\hline & NA & 0 & 0 & 0 & 0 & 0 \\
\hline & Total & 16 & 3 & 15 & 0 & 34 \\
\hline \multirow{5}{*}{26} & High & 11 & 1 & 11 & 0 & 23 \\
\hline & Low & 0 & 3 & 0 & 0 & 3 \\
\hline & Medium & 0 & 0 & 8 & 0 & 8 \\
\hline & NA & 0 & 0 & 0 & 0 & 0 \\
\hline & Total & 11 & 4 & 19 & 0 & 34 \\
\hline \multirow[t]{5}{*}{27} & High & 10 & 1 & 10 & 0 & 21 \\
\hline & Low & 0 & 4 & 0 & 0 & 4 \\
\hline & Medium & 1 & 1 & 7 & 0 & 9 \\
\hline & NA & 0 & 0 & 0 & 0 & 0 \\
\hline & Total & 11 & 6 & 17 & 0 & 34 \\
\hline \multirow[t]{5}{*}{28} & High & 13 & 2 & 10 & 0 & 25 \\
\hline & Low & 0 & 4 & 0 & 0 & 4 \\
\hline & Medium & 1 & 0 & 4 & 0 & 5 \\
\hline & NA & 0 & 0 & 0 & 0 & 0 \\
\hline & Total & 14 & 6 & 14 & 0 & 34 \\
\hline \multirow[t]{5}{*}{29} & High & 18 & 1 & 4 & 0 & 23 \\
\hline & Low & 0 & 1 & 0 & 0 & 1 \\
\hline & Medium & 1 & 0 & 9 & 0 & 10 \\
\hline & NA & 0 & 0 & 0 & 0 & 0 \\
\hline & Total & 19 & 2 & 13 & 0 & 34 \\
\hline \multirow[t]{4}{*}{30} & High & 15 & 3 & 11 & 0 & 29 \\
\hline & Low & 0 & 1 & 0 & 0 & 1 \\
\hline & Medium & 0 & 0 & 4 & 0 & 4 \\
\hline & NA & 0 & 0 & 0 & 0 & 0 \\
\hline
\end{tabular}

\begin{tabular}{|c|c|c|c|c|c|c|}
\hline \multirow{2}{*}{$\begin{array}{l}\text { Questio } \\
\mathrm{n}\end{array}$} & \multirow{2}{*}{ Importance } & \multicolumn{4}{|c|}{ Performance } & \multirow{2}{*}{ Total } \\
\hline & & High & Low & Medium & NA & \\
\hline \multirow[t]{2}{*}{ (1) } & (2) & (3) & (4) & (5) & (6) & (7) \\
\hline & Total & 15 & 4 & 15 & 0 & 34 \\
\hline \multirow[t]{5}{*}{31} & High & 17 & 0 & 10 & 0 & 27 \\
\hline & Low & 0 & 2 & 0 & 0 & 2 \\
\hline & Medium & 1 & 0 & 4 & 0 & 5 \\
\hline & NA & 0 & 0 & 0 & 0 & 0 \\
\hline & Total & 18 & 2 & 14 & 0 & 34 \\
\hline \multirow[t]{5}{*}{32} & High & 16 & 0 & 7 & 0 & 23 \\
\hline & Low & 0 & 2 & 0 & 0 & 2 \\
\hline & Medium & 0 & 0 & 9 & 0 & 9 \\
\hline & NA & 0 & 0 & 0 & 0 & 0 \\
\hline & Total & 16 & 2 & 16 & 0 & 34 \\
\hline \multirow[t]{5}{*}{33} & High & 5 & 0 & 17 & 0 & 22 \\
\hline & Low & 0 & 3 & 0 & 0 & 3 \\
\hline & Medium & 1 & 0 & 8 & 0 & 9 \\
\hline & NA & 0 & 0 & 0 & 0 & 0 \\
\hline & Total & 6 & 3 & 25 & 0 & 34 \\
\hline \multirow[t]{5}{*}{34} & High & 7 & 0 & 11 & 0 & 18 \\
\hline & Low & 0 & 4 & 1 & 0 & 5 \\
\hline & Medium & 1 & 0 & 10 & 0 & 11 \\
\hline & NA & 0 & 0 & 0 & 0 & 0 \\
\hline & Total & 8 & 4 & 22 & 0 & 34 \\
\hline
\end{tabular}

The attainment Index (W) is calculated as

$$
W=\frac{1}{w_{11} N} \sum_{i=1}^{m} \sum_{j=1}^{n} w_{i j} a_{i j},
$$

Where $a_{i j}$ the entries in the response matrix $w_{i j j}$, are the weighting co-efficients. In the present study it is , $m=n=3$. $w_{11}$ Typically, denotes the largest weight, and in this case, it is also literally so.

While the maximum permissible weighted value would have been 340 (sample size 34 and maximum weight 10), the minimum would be -170 , as the lowest weight is -5 .

The scores are standardized to have them not to exceed 1 and the minimum is allowed to be negative. Thus, all the matrix totals, correspondingto each question, are divided by 340 . The minimum would therefore, not fall below -0.5 , signifying the rock bottom.

Highest level of attainment on this index would indicate "perfect service" by the convention centre. However, this is hardly possible to achieve. In fact, each Convention Centre would have some specific strengths, which may not be possible for the others to replicate or challenge. Thus, there would be no "uniformly inferior" convention centre among those picked up for comparison. The idea would be to look for such a centre that has, in the overall, a high score, and is

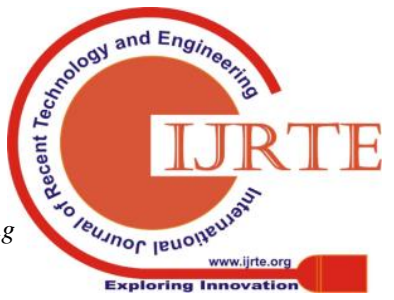


able to meet the requirements of the clients most of the attributes that are usually sought after.

. The Responses in the categories in the final data analysis is given below with the score calculated. The Importance Performance Analysis was applied to thirty four different questions.

Table 7.The Scores per attribute are as follows:

\begin{tabular}{|c|c|c|}
\hline $\begin{array}{l}\text { Attrib } \\
\text { ute }\end{array}$ & Description & Score \\
\hline 1 & $\begin{array}{l}\text { Visa, immigration clearances and } \\
\text { other regulatory issues for travel to } \\
\text { India }\end{array}$ & 0.29 \\
\hline 2 & $\begin{array}{l}\text { Air Connectivity from your usual } \\
\text { place of work to the current event } \\
\text { venue city }\end{array}$ & 0.29 \\
\hline 3 & $\begin{array}{l}\text { Ship from your usual place of } \\
\text { work to India, and from there to } \\
\text { current event venue city by air, } \\
\text { road or train }\end{array}$ & 0.27 \\
\hline 4 & $\begin{array}{l}\text { Facilitation of onward travel from } \\
\text { the current event venue city } \\
\text { (including ticketing, FOREX, } \\
\text { airport drop etc.) }\end{array}$ & 0.26 \\
\hline 5 & $\begin{array}{l}\text { Facilitation of travel to the current } \\
\text { event venue city }\end{array}$ & 0.57 \\
\hline 6 & $\begin{array}{l}\text { Accommodation facilities (room/ } \\
\text { suite) }\end{array}$ & 0.66 \\
\hline 7 & 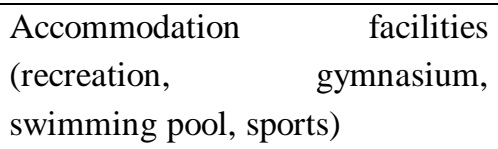 & 0.38 \\
\hline 8 & $\begin{array}{l}\text { Facilitation of local Travel within } \\
\text { city of current event : travel to and } \\
\text { fro event venue }\end{array}$ & 0.28 \\
\hline 9 & $\begin{array}{l}\text { Facilitation of other local Travel } \\
\text { within city of current event (car/ } \\
\text { taxi/ road/ train/ others) }\end{array}$ & 0.32 \\
\hline 10 & $\begin{array}{l}\text { Local Travel within city of current } \\
\text { event (traffic situation } \\
\text { cumbersome traffic scores low) }\end{array}$ & 0.29 \\
\hline 11 & $\begin{array}{l}\text { Facilitation of Sightseeing } \\
\text { (information and access) }\end{array}$ & 0.35 \\
\hline 12 & $\begin{array}{l}\text { Quality of Sightseeing : Places } \\
\text { visited }\end{array}$ & 0.52 \\
\hline 13 & $\begin{array}{l}\text { Facilitation of other entertainment } \\
\text { in city (information and access) }\end{array}$ & 0.41 \\
\hline 14 & $\begin{array}{l}\text { Quality of other recreation/ } \\
\text { entertainment in city }\end{array}$ & 0.28 \\
\hline 15 & Souvenirs (Local handicrafts) & 0.39 \\
\hline 16 & $\begin{array}{l}\text { Souvenirs (other than Local } \\
\text { handicrafts) }\end{array}$ & 0.43 \\
\hline 17 & Cordiality in interaction with local & 0.43 \\
\hline
\end{tabular}

\begin{tabular}{|c|c|c|}
\hline & population & \\
\hline 18 & $\begin{array}{l}\text { Regulatory issues pertaining to } \\
\text { hosting the event (which might } \\
\text { have been notified by the } \\
\text { organizer, or observed by delays or } \\
\text { post-ponements) [Obstructions } \\
\text { observed score high, nevertheless] }\end{array}$ & 0.35 \\
\hline 19 & $\begin{array}{l}\text { Pre-event } \begin{array}{l}\text { management } \\
\text { (organizational preparedness as } \\
\text { observed) }\end{array} \\
\end{array}$ & 0.57 \\
\hline 20 & Pre-event gathering/ dinner & 0.61 \\
\hline 21 & $\begin{array}{l}\text { Quality of skilled manpower } \\
\text { handling the event }\end{array}$ & 0.29 \\
\hline 22 & $\begin{array}{l}\text { Facilitation of Registration for } \\
\text { event : Single window or } \\
\text { web-based (hassle-free is 'High') }\end{array}$ & 0.57 \\
\hline 23 & $\begin{array}{l}\text { Equipment support at the Venue } \\
\text { (multi-media for presentation, } \\
\text { etc.) }\end{array}$ & 0.62 \\
\hline 24 & $\begin{array}{l}\text { Other equipment support at the } \\
\text { Venue }\end{array}$ & 0.40 \\
\hline 25 & $\begin{array}{lrr}\text { Catering/ } & \text { working } & \text { lunch/ } \\
\text { refreshments } & \text { during } & \text { event/ } \\
\text { session } & & \end{array}$ & 0.47 \\
\hline 26 & $\begin{array}{l}\text { Catering/ refreshments outside } \\
\text { session, within the venue of } \\
\text { current event }\end{array}$ & 0.38 \\
\hline 27 & $\begin{array}{l}\text { Catering/ restaurants outside the } \\
\text { venue of current event, within city }\end{array}$ & 0.35 \\
\hline 28 & Aspects in event venue: Aesthetics & 0.40 \\
\hline 29 & Aspects in event venue: Ambience & 0.63 \\
\hline 30 & Aspects in event venue: Hygiene & 0.41 \\
\hline 31 & $\begin{array}{l}\text { Aspects in event venue: } \\
\text { Air-conditioning }\end{array}$ & 0.54 \\
\hline 32 & $\begin{array}{l}\text { Aspects in event venue: Other } \\
\text { Infrastructure }\end{array}$ & 0.56 \\
\hline 33 & $\begin{array}{l}\text { Observation about political } \\
\text { stability in India }\end{array}$ & 0.21 \\
\hline 34 & $\begin{array}{l}\text { Observation about economic } \\
\text { stability in India }\end{array}$ & 0.32 \\
\hline
\end{tabular}

The maximum score observed is 0.66 for Attribute 6 ("Accommodation facilities (room/ suite)"), while the minimum is 0.21 , observed in the case of attribute 33 ("Observation about political stability in India"). 
Table 8.The average score is 0.42 of the 34 attributes, scores of as many as 18 attributes have remained within one sigma $(0.18)$ of the mean $(0.42)$. These are:

\begin{tabular}{|c|c|c|}
\hline $\begin{array}{l}\text { Attrib } \\
\text { ute }\end{array}$ & Description & Score \\
\hline 7 & $\begin{array}{l}\text { Accommodation facilities } \\
\text { (recreation, gymnasium, swimming } \\
\text { pool, sports) }\end{array}$ & 0.38 \\
\hline 9 & $\begin{array}{l}\text { Facilitation of other local Travel } \\
\text { within city of current event (car/ } \\
\text { taxi/ road/ train/ others) }\end{array}$ & 0.32 \\
\hline 11 & $\begin{array}{l}\text { Facilitation of Sightseeing } \\
\text { (information and access) }\end{array}$ & 0.35 \\
\hline 12 & $\begin{array}{l}\text { Quality of Sightseeing : Places } \\
\text { visited }\end{array}$ & 0.52 \\
\hline 13 & $\begin{array}{l}\text { Facilitation of other entertainment } \\
\text { in city (information and access) }\end{array}$ & 0.41 \\
\hline 15 & Souvenirs (Local handicrafts) & 0.39 \\
\hline 16 & $\begin{array}{l}\text { Souvenirs (other than Local } \\
\text { handicrafts) }\end{array}$ & 0.43 \\
\hline 17 & $\begin{array}{l}\text { Cordiality in interaction with local } \\
\text { population }\end{array}$ & 0.43 \\
\hline 18 & $\begin{array}{l}\text { Regulatory issues pertaining to } \\
\text { hosting the event (which might have } \\
\text { been notified by the organizer, or } \\
\text { observed by delays or } \\
\text { post-ponements) [Obstructions } \\
\text { observed score high, nevertheless] }\end{array}$ & 0.35 \\
\hline 21 & $\begin{array}{l}\text { Quality of skilled manpower } \\
\text { handling the event }\end{array}$ & 0.29 \\
\hline 24 & $\begin{array}{l}\text { Other equipment support at the } \\
\text { Venue }\end{array}$ & 0.40 \\
\hline 25 & $\begin{array}{l}\text { Catering/ working lunch/ } \\
\text { refreshments during event/ session }\end{array}$ & 0.47 \\
\hline 26 & $\begin{array}{l}\text { Catering/ refreshments outside } \\
\text { session, within the venue of current } \\
\text { event }\end{array}$ & 0.38 \\
\hline 27 & $\begin{array}{l}\text { Catering/ restaurants outside the } \\
\text { venue of current event, within city }\end{array}$ & 0.35 \\
\hline 28 & Aspects in event venue: Aesthetics & 0.40 \\
\hline 30 & Aspects in event venue: Hygiene & 0.41 \\
\hline 31 & $\begin{array}{l}\text { Aspects in event venue: } \\
\text { Air-conditioning }\end{array}$ & 0.54 \\
\hline 34 & $\begin{array}{l}\text { Observation about economic } \\
\text { stability in India }\end{array}$ & 0.32 \\
\hline
\end{tabular}

To measure the quality of the services provided by the Convention Centers to the target audience, a long list of were attributes were selected, without claiming to be comprehensive. The scoring also shows that there is considerable variation in perception in each of the attributes. In other words, none of the attributes chosen was too commonplace, inviting any stock answer of affirmation or negation.

The weighted scores have also shown that the levels of disapproval observed (distance between expectation measured in terms of the importance attached and the service actually received, in terms of performance) has been quite varied, but none of them has been below 0.2 nor above 0.8 .It may be noted that $\mathrm{R}$ software sorts by alphabetical Order, hence High, Low, Medium, is not applicable.For the purpose of analysis of the above tabulated figures, the following criteria were used for assigning weights for each cell:For an item with Importance Level "High",

Actual Performance of High gets a value of 10, Medium gets a -1 , while Low gets -5 .

For an item with Importance Level "Medium",

Actual Performance of High gets a value of 5, Medium gets a 4, while Low gets -2 .

For an item with Importance Level "Low",

Actual Performance of High gets a value of 4, Medium gets a 2, while Low gets 1 .

"NotApplicable"entries, were ignored by assigning the value zero.

\section{CONCLUSION}

The Weighting scheme assigns highest weight to an aspect assessed to be of high importance and to have been met with well at the venue. Any item with higher level of perceived importance but the delivery of which is below par, gets a negative weight, and the farther the level of performance, the lower the weight. A par performance is assigned a positive weight, while an above-par performance gets a little more credit.

To measure the quality of the services provided by the Convention Centers to the target audience, 34 attributeswhich are comprehensive are selected. The scoring also shows that there is considerable variation in perception in each of the attributes. .

The weighted scores have also shown that the levels of disapproval observed (distance between expectation measured in terms of the importance attached and the service actually received, in terms of performance) has been quite varied, but none of them has been below 0.2 nor above 0.8 . Therefore, all the attributes are being examined. To measure the quality of the services provided by the Convention Centers to the target audience a list of nine attributes were selected Therefore it is concluded that of the three cities Hyderabad holds a competitive position compared to Delhi Mumbai and Kolkata. Apart from this it is observed that visa facilitation and air connectivity to the city is important and also performed well. The manpower at the venue did not perform well due to the lack of training and skill development. The courses which are widely prevalent are

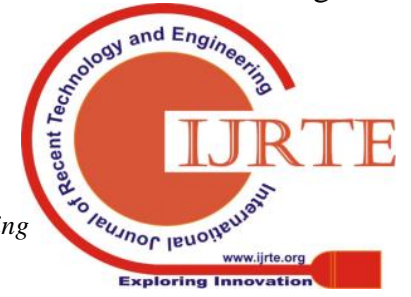




\section{Tangible and Intangible Attributes for the Development of Convention Tourism - An Economic Proposition}

only engineering and medicine. Tourism education imparting colleges nor Universities are present in the complete State. There is huge requirement of Manpower Training to handle the Convention Tourism activities in the state of Telangana..Accommodation facilities and Foreign Exchange transaction performed well which were essential for business traveler. The facilitation of local travel was important and performed well in Mumbai and Delhi but in Hyderabad due to excessive traffic it could not perform well. Hence it is observed that Hyderabad holds a competitive edge in seven attributes compare to Delhi and Mumbai.

\section{REFERENCES}

[1] Braun, B. M., \& Rungeling, B. (1992). The relative economic impact of convention and tourist visitors on a regional economy: a case study. International Journal of Hospitality Management, 11(1), 65-71.

[2] Kim, S. S., Chon, K., \& Chung, K. Y. (2003). Convention industry in South Korea: an economic impact analysis. Tourism Management, 24(5), 533-541.

[3] Braun, B. M. (1992). The economic contribution of conventions: The case of Orlando, Florida. Journal of Travel Research, 30(3), 32-37.

[4] Yoo, J. J. E., \& Weber, K. (2005). Progress in convention tourism research. Journal of Hospitality \& Tourism Research, 29(2), 194-222.

[5] Dwyer, L., Mellor, R., Mistilis, N., \& Mules, T. (2000). A framework for assessing "tangible" and "intangible" impacts of events and conventions. Event management, 6(3), 175-189.

[6] Mackellar, J. (2007, January). Conventions, festivals, and tourism: Exploring the network that binds. In Journal of Convention \& Event Tourism (Vol. 8, No. 2, pp. 45-56). Taylor \& Francis Group

[7] McCartney, G. (2008, November). The CAT (casino tourism) and the MICE (meetings, incentives, conventions, exhibitions): Key development considerations for the convention and exhibition industry in Macao. In Journal of Convention \& Event Tourism (Vol. 9, No. 4, pp. 293-308). Taylor \& Francis Group.

[8] Weber, K. (2001). Meeting planners' use and evaluation of convention and visitor bureaus. Tourism Management, 22(6), 599-606.

[9] . J. E., \& Chon, K. (2008). Factors affecting convention participation decision-making: Developing a measurement scale. Journal of Travel Research.

[10] Weber, K., \& Roehl, W. S. (2001, August). Service quality issues for convention and visitor bureaus. In Journal of Convention \& Exhibition Management (Vol. 3, No. 1, pp. 1-19). Taylor \& Francis Group.

[11] Mair, J., \& Jago, L. (2010). The development of a conceptual model of greening in the business events tourism sector. Journal of Sustainable Tourism, 18(1), 77-94.

[12] Lee, M. J., \& Back, K. J. (2007). Effects of destination image on meeting participation intentions: Empirical findings from a professional association and its annual convention. The Service Industries Journal, 27(1), 59-73.

[13] Yoo, J. J. E., \& Zhao, X. (2010). Revisiting determinants of convention participation decision making. Journal of Travel \& Tourism Marketing, 27(2), 179-192.

[14] Breiter, D., \& Milman, A. (2006). Attendees' needs and service priorities in a large convention center: Application of the importance-performance theory.Tourism Management, 27(6), 1364-1370.

[15] Go, F., \& Zhang, W. (1997). Applying importance-performance analysis to Beijing as an international meeting destination. Journal of Travel Research,35(4), 42-49.

[16] Ennew, C. T., Reed, G. V., \& Binks, M. R. (1993). Importance-performance analysis and the measurement of service quality. European journal of marketing, 27(2), 59-70.

[17] Zhang, H. Q., Leung, V., \& Qu, H. (2007). A refined model of factors affecting convention participation decision-making. Tourism Management, 28(4), 1123-1127.

[18] Sivakoti Reddy, M. (2019). Impact of RSERVQUAL on customer satisfaction: A comparative analysis between traditional and multi-channel retailing. International Journal of Recent Technology and Engineering. 8(1), pp. 2917-2920.

[19] Sivakoti Reddy, M., Venkateswarlu, N.(2019). Customer relationship management practices and their impact over customer purchase decisions: A study on the selected private sector banks housing finance schemes. International Journal of Innovative Technology and Exploring Engineering. 8(7), pp. 1720-1728.
[20] Sivakoti Reddy, M., Murali Krishna, S.M.(2019). Influential role of retail service quality in food and grocery retailing: A comparative study between traditional and multi-channel retailing. International Journal of Management and Business Research. 9(2), pp. 68-73.

[21] Sivakoti Reddy, M., Naga Bhaskar, M., Nagabhushan, A. (2016). Saga of silicon plate: An empirical analysis on the impact of socio economic factors of farmers on inception of solar plants. International Journal of Control Theory and Applications. 9(29), pp. 257-266.

[22] Manukonda et al. (2019). What Motivates Students To Attend Guest Lectures?. The International Journal of Learning in Higher Education. Volume 26, Issue 1. 23-34.

[23] Hymavathi, C.H., Koneru, K.(2019). Investors perception towards Indian commodity market: An empirical analysis with reference to Amaravathi region of Andhra Pradesh. International Journal of Innovative Technology and Exploring Engineering. 8(7), pp. 1708-1714.

[24] Neelima, J., Koneru, K.(2019). Assessing the role of organizational culture in determining the employee performance - empirical evidence from Indian pharmaceutical sector. International Journal of Innovative Technology and Exploring Engineering. 8(7), pp. 1701-1707.

[25] Kishan Varma, M.S., Koneru, K., Yedukondalu, D.(2019). Affect of worksite wellness interventions towards occupational stress. International Journal of Recent Technology and Engineering. 8(1), pp. 2874-2879.

[26] Hymavathi, C., Koneru, K. (2019). Role of perceived risk in mutual funds selection behavior: An analysis among the selected mutual fund investors. International Journal of Engineering and Advanced Technology. 8(4), pp 1913-1920.

[27] Suhasini, T., Koneru, K. (2019). Employee engagement through HRD practices on employee satisfaction and employee loyalty: An empirical evidence from Indian IT industry. International Journal of Engineering and Advanced Technology. 8(4), pp 1788-1794.

[28] Suhasini, T. Koneru, K. (2018). A study on employee engagement driving factors and their impact over employee satisfaction - An empirical evidence from Indian it industry. International Journal of Mechanical Engineering and Technology. 9(4), pp. 725-732

[29] Hymavathi, C.H., Koneru, K.(2018). Investors' awareness towards commodities market with reference to GUNTUR city, Andhra Pradesh. International Journal of Engineering and Technology(UAE). 7(2), pp 1104-1106 